\title{
Universality in Measure in the Bulk for Varying Weights
}

\section{ELI LEVIN \& Doron S. LUBINSKY}

AвSTRACT. We prove that universality holds in measure for sequences $\left\{g_{n} e^{-2 n Q}\right\}$ of varying weights, where $e^{-Q}$ is an exponential weight, and the functions $\left\{g_{n}\right\}$ admit suitable polynomial approximations.

\section{Introduction}

In the theory of random Hermitian matrices, one considers a probability distribution $\mathcal{P}^{(n)}$ on the eigenvalues $x_{1} \leq x_{2} \leq \cdots \leq x_{n}$ of $n \times n$ Hermitian matrices,

$$
\mathcal{P}^{(n)}\left(x_{1}, x_{2}, \ldots, x_{n}\right)=c e^{-\sum_{j=1}^{n} 2 n Q_{n}\left(x_{j}\right)} \prod_{i<j}\left(x_{i}-x_{j}\right)^{2} .
$$

See [5, p. $106 \mathrm{ff}$.]. Here, $c$ is a normalizing constant, often called the partition function, and $Q_{n}$ is a given function. In the Gaussian unitary ensemble, $Q_{n}(x)=$ $\frac{1}{2} x^{2}$.

Orthogonal polynomials play a crucial role in analyzing these. For $n \geq 1$, let $\mu_{n}$ be a finite positive Borel measure with support supp $\left[\mu_{n}\right]$ containing infinitely many points. We may define orthonormal polynomials

$$
p_{m}\left(\mu_{n}, x\right)=\gamma_{m}\left(\mu_{n}\right) x^{m}+\cdots, \quad \gamma_{m}\left(\mu_{n}\right)>0,
$$

$m=0,1,2, \ldots$, satisfying the orthonormality conditions

$$
\int p_{k}\left(\mu_{n}, x\right) p_{\ell}\left(\mu_{n}, x\right) d \mu_{n}(x)=\delta_{k \ell}
$$

Throughout we use $\mu_{n}^{\prime}$ to denote the Radon-Nikodym derivative of $\mu_{n}$. The $n$th reproducing kernel for $\mu_{n}$ is [10;23]

$$
K_{n}\left(\mu_{n}, x, y\right)=\sum_{k=0}^{n-1} p_{k}\left(\mu_{n}, x\right) p_{k}\left(\mu_{n}, y\right),
$$

and the normalized kernel is

$$
\tilde{K}_{n}\left(\mu_{n}, x, y\right)=\mu_{n}^{\prime}(x)^{1 / 2} \mu_{n}^{\prime}(y)^{1 / 2} K_{n}\left(\mu_{n}, x, y\right) .
$$

The $n$th Christoffel function is

$$
\lambda_{n}\left(\mu_{n}, x\right)=K_{n}\left(\mu_{n}, x, x\right)^{-1} .
$$

When

$$
d \mu_{n}(x)=e^{-2 n Q_{n}(x)} d x
$$

Received July 3, 2015. Revision received October 19, 2016.

Second author's research supported by NSF grant DMS136208. 
there is the basic formula for the probability distribution $\mathcal{P}^{(n)}[5$, p. 112]:

$$
\mathcal{P}^{(n)}\left(x_{1}, x_{2}, \ldots, x_{n}\right)=\frac{1}{n !} \operatorname{det}\left(\tilde{K}_{n}\left(\mu_{n}, x_{i}, x_{j}\right)\right)_{1 \leq i, j \leq n} .
$$

Note that this is the standard definition, which includes the factor $\mu_{n}^{\prime}\left(x_{1}\right) \mu_{n}^{\prime}\left(x_{2}\right)$. $\cdots \mu_{n}^{\prime}\left(x_{n}\right)$ in the right-hand side. One particularly important quantity is the $m$ point correlation function,

$$
\begin{aligned}
& R_{m}\left(\mu_{n}, x_{1}, x_{2}, \ldots, x_{m}\right) \\
& \quad=\frac{n !}{(n-m) !} \int \cdots \int \mathcal{P}^{(n)}\left(x_{1}, x_{2}, \ldots, x_{n}\right) d x_{m+1} d x_{m+2} \cdots d x_{n} .
\end{aligned}
$$

The function $R_{m}$ admits the remarkable identity [5, p. 112], first proved by Freeman Dyson,

$$
R_{m}\left(\mu_{n}, x_{1}, x_{2}, \ldots, x_{m}\right)=\operatorname{det}\left(\tilde{K}_{n}\left(\mu_{n}, x_{i}, x_{j}\right)\right)_{1 \leq i, j \leq m} .
$$

The universality limit in the bulk asserts that, for fixed $m \geq 2$, $\xi$ in a suitable subset of the (common) supports of $\left\{\mu_{n}\right\}$, and real $a_{1}, a_{2}, \ldots, a_{m}$, we have

$$
\begin{aligned}
\lim _{n \rightarrow \infty} & \frac{1}{\tilde{K}_{n}\left(\mu_{n}, \xi, \xi\right)^{m}} R_{m}\left(\mu_{n}, \xi+\frac{a_{1}}{\tilde{K}_{n}\left(\mu_{n}, \xi, \xi\right)}, \xi+\frac{a_{2}}{\tilde{K}_{n}\left(\mu_{n}, \xi, \xi\right)}, \ldots,\right. \\
& \left.\xi+\frac{a_{m}}{\tilde{K}_{n}\left(\mu_{n}, \xi, \xi\right)}\right) \\
= & \operatorname{det}\left(\mathbb{S}\left(a_{i}-a_{j}\right)\right)_{1 \leq i, j \leq m}
\end{aligned}
$$

where

$$
\mathbb{S}(t)=\frac{\sin \pi t}{\pi t}
$$

is the sinc kernel. Because $m$ is fixed in this limit, this reduces to the case $m=2$, namely

$$
\lim _{n \rightarrow \infty} \frac{\tilde{K}_{n}\left(\mu_{n}, \xi+\frac{a}{\tilde{K}_{n}\left(\mu_{n}, \xi, \xi\right)}, \xi+\frac{b}{\tilde{K}_{n}\left(\mu_{n}, \xi, \xi\right)}\right)}{\tilde{K}_{n}\left(\mu_{n}, \xi, \xi\right)}=\mathbb{S}(a-b) .
$$

Typically, this is established uniformly for $a, b$ in compact subsets of the real line. Thus, an assertion about the distribution of eigenvalues of random matrices has been reduced to a technical limit involving orthogonal polynomials. See $[1 ; 2$; $4 ; 5 ; 6 ; 8 ; 9 ; 13 ; 15 ; 16 ; 17 ; 18 ; 19 ; 23 ; 24 ; 27 ; 28]$ for some references to the extensive literature on this topic.

We shall need some concepts from potential theory for external fields [22]. Let $\Sigma$ be a closed set on the real line, and $W(x)=\exp (-Q(x))$ be an upper semicontinuous function on $\Sigma$ that is positive on a set of positive linear Lebesgue measure. If $\Sigma$ is unbounded, then we assume that

$$
\lim _{|x| \rightarrow \infty, x \in \Sigma} W(x)|x|=0 .
$$


We say, following Saff and Totik, [22], that $W$ is admissible. Associated with $\Sigma$ and $Q$, we may consider the extremal problem

$$
\inf _{v}\left(\iint \log \frac{1}{|x-t|} d v(x) d v(t)+2 \int Q d v\right),
$$

where the inf is taken over all positive Borel measures $v$ with support in $\Sigma$ and $v(\Sigma)=1$. The inf is attained by a unique equilibrium measure $\omega_{Q}$, characterized by the following conditions: let

$$
U^{\omega_{Q}}(z)=\int \log \frac{1}{|z-t|} d \omega_{Q}(t)
$$

denote the logarithmic potential for $\omega_{Q}$, and let

$$
\mathcal{S}_{Q}=\operatorname{supp}\left[\omega_{Q}\right]
$$

denote the (compact) support of the equilibrium measure. Then [22, Theorem I.1.3, p. 27]

$$
\begin{array}{ll}
U^{\omega_{Q}}+Q \geq F_{Q} & \text { q.e. on } \Sigma \\
U^{\omega_{Q}}+Q=F_{Q} & \text { q.e. in } \mathcal{S}_{Q} .
\end{array}
$$

Here the number $F_{Q}$ is a constant, and q.e. stands for quasi-everywhere, that is, except on a set of capacity 0 . Notice that we are using $\omega_{Q}$ for the equilibrium measure, rather than the more standard $\mu_{W}$ or $\nu_{W}$, to avoid confusion with $\mu_{n}$ or $v_{n}$.

Our first result in $[15$, p. 747 , Theorem 1.1] was the following. Its proof depended heavily on asymptotics of Christoffel functions that has been established by Vili Totik [26]:

THeOREM A. Let $W=e^{-Q}$ be a continuous nonnegative function on the set $\Sigma$, which is assumed to consist of at most finitely many intervals. If $\Sigma$ is unbounded, then we also assume (1.4). Let $h$ be a bounded positive continuous function on $\Sigma$, and for $n \geq 1$, let

$$
d \mu_{n}(x)=\left(h W^{2 n}\right)(x) d x .
$$

Let $J$ be a closed interval lying in the interior of $\mathcal{S}_{Q}=\operatorname{supp}\left[\omega_{Q}\right]$, where $\omega_{Q}$ denotes the equilibrium measure for $W$. Assume that $\omega_{Q}$ is absolutely continuous in a neighborhood of $J$ and that $\omega_{Q}^{\prime}$ and $Q^{\prime}$ are continuous in that neighborhood with $\omega_{Q}^{\prime}>0$ there. Then, uniformly for $\xi \in J$ and $a, b$ in compact subsets of the real line, we have

$$
\lim _{n \rightarrow \infty} \frac{\tilde{K}_{n}\left(\mu_{n}, \xi+\frac{a}{\tilde{K}_{n}\left(\mu_{n}, \xi, \xi\right)}, \xi+\frac{b}{\tilde{K}_{n}\left(\mu_{n}, \xi, \xi\right)}\right)}{\tilde{K}_{n}\left(\mu_{n}, \xi, \xi\right)}=\mathbb{S}(a-b) .
$$

In particular, if $Q^{\prime}$ satisfies a Lipschitz condition of some positive order in a neighborhood of $J$, then [22, p. 199] $\omega_{Q}^{\prime}$, is continuous there, and hence we obtain universality except near zeros of $\omega_{Q}^{\prime}$. Note too that when $Q$ is convex in $\Sigma$, or $x Q^{\prime}(x)$ is increasing there, the support of $\omega_{Q}$ consists of at most finitely many intervals, with at most one interval per component of $\Sigma$ [22, p. 199]. 
Theorem A is a particular case of a more general result in $[15, \mathrm{p} .748$, Theorem 1.2], which allowed fairly general sequences of weights, but involved hypotheses on asymptotics of ratios of their Christoffel functions. In [15], applications were also made to universality for fixed exponential weights on the real line.

Our goal in this paper is to prove that universality holds in measure for varying weights under more general hypotheses than those in Theorem A. Our first result is an equiconvergence result. Throughout, meas denotes the linear Lebesgue measure. We also say that a sequence of real-valued functions $\left\{f_{n}\right\}$ defined on a compact set $\Sigma$ converges in measure to a function $g$ if for each $\varepsilon>0$,

$$
\operatorname{meas}\left\{x \in \Sigma:\left|f_{n}(x)-g(x)\right|>\varepsilon\right\} \rightarrow 0 \quad \text { as } n \rightarrow \infty .
$$

THeOREM 1.1. Let $\Sigma$ be a closed set, and $\left\{v_{n}\right\}$ and $\left\{\mu_{n}\right\}$ be sequences of positive measures on $\Sigma$. Assume that, for $n \geq 1$,

$$
\mu_{n}^{\prime}(x)=h(x) e^{-2 n Q(x)}, \quad x \in \Sigma,
$$

where $h$ is bounded, positive, and continuous on $\Sigma$, and $e^{-Q}$ is admissible, and also, as $n \rightarrow \infty$,

$$
\frac{1}{n} K_{n}\left(\mu_{n}, x, x\right) \mu_{n}^{\prime}(x) \rightarrow \omega_{Q}^{\prime} \quad \text { in measure in } \mathcal{S}_{Q} .
$$

Assume also that, for $n \geq 1$,

$$
v_{n}^{\prime}(x)=g_{n}(x) \mu_{n}^{\prime}(x), \quad x \in \Sigma,
$$

and for $n \geq 1$, there exists a polynomial $S_{n}$ of degree $\ell_{n}$ that is positive in $\Sigma$, with

$$
\ell_{n}=o(n), \quad n \rightarrow \infty,
$$

and

while as $n \rightarrow \infty$,

$$
S_{n}^{2}(x) g_{n}(x) \leq 1, \quad \text { a.e. } x \in \Sigma,
$$

$$
S_{n}^{2} g_{n} \rightarrow 1 \text { in measure in } \mathcal{S}_{Q} .
$$

Let $R, \varepsilon>0$. Then for large enough $n$, there exists a set $\mathcal{E}_{n}$ of linear Lebesgue measure $<\varepsilon$ such that

$$
\begin{aligned}
& \sup _{\substack{|a|,|b| \leq R, \leq \\
\xi \in \mathcal{S}_{Q} \backslash \mathcal{E}_{n}}} \frac{1}{n} \mid K_{n}\left(v_{n}, \xi+\frac{a}{n}, \xi+\frac{b}{n}\right) \\
& \quad-S_{n}\left(\xi+\frac{a}{n}\right) S_{n}\left(\xi+\frac{b}{n}\right) K_{n}\left(\mu_{n}, \xi+\frac{a}{n}, \xi+\frac{b}{n}\right) \mid v_{n}^{\prime}(\xi)=o(1) .
\end{aligned}
$$

The set $\mathcal{E}_{n}$ does not depend on $R$.

REMARKs. (a) Note that in (1.14), $a, b$ are complex numbers.

(b) In (1.9), we could equivalently assume that the convergence in measure takes place in $\Sigma$.

(c) Note that (1.9) holds under the conditions of Theorem A; see [26].

(d) A more explicit form of Theorem 1.1 is given in Theorem 4.3. 
(e) The conclusion of Theorem 1.1 is in fact almost uniform convergence, as used in Egorov's theorem, and so is a little stronger than convergence in measure.

One case where we can find suitable approximating polynomials $\left\{S_{n}\right\}$ is in the following result, where $\Sigma$ is assumed to be a union of finitely many compact intervals. Let $\alpha \in(0, \infty)$, and for $n \geq 1$, let $\varphi_{n}: \Sigma \rightarrow \mathbb{R}$. We say that $\left\{\varphi_{n}\right\}$ is uniformly smooth of order $\alpha$ if

(i) when $\alpha=k$, where $k$ is a positive integer, $\varphi_{n}^{(k-1)}$ is absolutely continuous for each $n \geq 1$, with

$$
\sup _{n}\left\|\varphi_{n}^{(k)}\right\|_{L_{\infty}(\Sigma)}<\infty
$$

(ii) when $\alpha=k+\Delta$, where $k$ is a nonnegative integer, and $0<\Delta<1, \varphi_{n}^{(k)}$ satisfies a uniform Lipschitz condition of order $\Delta$ :

$$
\sup _{n} \sup _{x, y \in \Sigma} \frac{\left|\varphi_{n}^{(k)}(x)-\varphi_{n}^{(k)}(y)\right|}{|x-y|^{\Delta}}<\infty \text {. }
$$

THEOREM 1.2. Let $\Sigma$ consist of finitely many compact intervals, and let

$$
v_{n}^{\prime}(x)=h(x) e^{\tau_{n} \varphi_{n}(x)}\left(\prod_{j=1}^{N}\left|x-b_{n j}\right|^{\beta_{n j}}\right)^{n} e^{-2 n Q(x)}, \quad x \in \Sigma,
$$

where $h$ is a positive continuous function on $\Sigma$, and where $Q$ is continuous, while $Q^{\prime}$ is continuous in $\Sigma$, except perhaps at finitely many points. Assume, moreover, that the equilibrium measure $\omega_{Q}$ is absolutely continuous and that $\omega_{Q}^{\prime}$ is positive and continuous in $\mathcal{S}_{Q}^{o}$, except perhaps at finitely many points. Assume also that $N \geq 1$ and all $\left\{b_{n j}\right\}_{n, j}$ lie in some compact subset of the real line, while

$$
\max _{1 \leq j \leq N} \beta_{n j} \rightarrow 0 \quad \text { as } n \rightarrow \infty .
$$

In addition, assume that $\alpha \in(0, \infty)$ and $\left\{\varphi_{n}\right\}$ is a sequence of real-valued functions on $\Sigma$ that is uniformly smooth of order $\alpha$, whereas for $n \geq 1$,

$$
\left\|\varphi_{n}\right\|_{L_{\infty}(\Sigma)} \leq 1
$$

and

$$
\tau_{n}=o\left(n^{\frac{\alpha}{1+\alpha}}\right), \quad n \rightarrow \infty
$$

Then, given $\varepsilon>0$,

$$
\begin{aligned}
& \operatorname{meas}\left\{x \in \mathcal{S}_{Q}: \sup _{|a|,|b| \leq R}\left|\frac{\tilde{K}_{n}\left(v_{n}, \xi+\frac{a}{n \omega_{Q}^{\prime}(\xi)}, \xi+\frac{b}{n \omega_{Q}^{\prime}(\xi)}\right)}{n \omega_{Q}^{\prime}(\xi)}-\mathbb{S}(a-b)\right|>\varepsilon\right\} \\
& \quad \rightarrow 0 \text { as } n \rightarrow \infty .
\end{aligned}
$$

Remarks. (a) Note that in (1.19), we restrict $x$ to lie in $\mathcal{S}_{Q}$ since we cannot have universality (at least in the sense above) outside this set. Moreover, $a, b$ are real since $\tilde{K}_{n}$ is only defined in $\Sigma$. 
(b) The requirement that $\Sigma$ is compact can be removed if we assume that the measures decay exponentially at $\infty$, allowing us to apply restricted range inequalities from, for example, [14;22].

(c) We could allow more general rates of growth of $\left\{\tau_{n}\right\}$ than (1.18). Moreover, if all $\left\{\varphi_{n}\right\}$ are analytic and uniformly bounded in an open set containing $\Sigma$ that is independent of $n$, we could replace (1.18) by

$$
\tau_{n}=o\left(\frac{n}{\log n}\right), \quad n \rightarrow \infty .
$$

(d) We could multiply by other factors, such as $e^{o(n) U^{\rho_{n}}}$, where $\left\{\rho_{n}\right\}$ are a sequence of measures supported on $\Sigma=[0,1]$ that admit polynomial approximation of the type described in [25, Theorem 8.1, p. 49].

\section{An Integral Estimate}

We start with the following simple comparison inequality.

Lemma 2.1. Let $m, n \geq 1$, and $\mu_{n}$ and $v_{n}$ be positive measures on the real line. Assume that $S_{n}$ is a polynomial of degree $\ell<n$ and

$$
n=m+\ell \text {. }
$$

Assume also that on $\mathbb{R}$,

$$
S_{n}^{2} d v_{n} \leq d \mu_{n}
$$

Then

$$
\begin{aligned}
\Gamma_{n} & :=\frac{1}{n} \iint\left(K_{n}\left(v_{n}, x, y\right)-S_{n}(x) S_{n}(y) K_{n}\left(\mu_{n}, x, y\right)\right)^{2} d v_{n}(x) d v_{n}(y) \\
& \leq 4\left(1+\frac{\ell}{n}-\frac{1}{n} \int S_{n}^{2}(y) K_{n}\left(\mu_{n}, y, y\right) d v_{n}(y)\right) .
\end{aligned}
$$

Proof. Using the reproducing kernel property and (2.1), followed by (2.2), we have

$$
\begin{aligned}
\Delta_{n}:= & \iint\left(K_{n}\left(v_{n}, x, y\right)-S_{n}(x) S_{n}(y) K_{m}\left(\mu_{n}, x, y\right)\right)^{2} d v_{n}(x) d v_{n}(y) \\
= & \iint K_{n}\left(v_{n}, x, y\right)^{2} d v_{n}(x) d v_{n}(y) \\
& -2 \int S_{n}(y)\left[\int S_{n}(x) K_{m}\left(\mu_{n}, x, y\right) K_{n}\left(v_{n}, x, y\right) d v_{n}(x)\right] d v_{n}(y) \\
& +\int S_{n}(y)^{2}\left[\int S_{n}(x)^{2} K_{m}\left(\mu_{n}, x, y\right)^{2} d v_{n}(x)\right] d v_{n}(y) \\
= & \int K_{n}\left(v_{n}, y, y\right) d v_{n}(y)-2 \int S_{n}^{2}(y) K_{m}\left(\mu_{n}, y, y\right) d v_{n}(y) \\
& +\int S_{n}(y)^{2}\left[\int S_{n}(x)^{2} K_{m}\left(\mu_{n}, x, y\right)^{2} d v_{n}(x)\right] d v_{n}(y)
\end{aligned}
$$




$$
\begin{aligned}
\leq & n-2 \int S_{n}^{2}(y) K_{m}\left(\mu_{n}, y, y\right) d v_{n}(y) \\
& +\iint K_{m}\left(\mu_{n}, x, y\right)^{2} d \mu_{n}(x) d \mu_{n}(y) \\
= & n+m-2 \int S_{n}^{2}(y) K_{m}\left(\mu_{n}, y, y\right) d v_{n}(y) .
\end{aligned}
$$

Next,

$$
\begin{aligned}
& \int S_{n}^{2}(y)\left(K_{n}-K_{m}\right)\left(\mu_{n}, y, y\right) d v_{n}(y) \\
& \quad \leq \int\left(K_{n}-K_{m}\right)\left(\mu_{n}, y, y\right) d \mu_{n}(y)=n-m .
\end{aligned}
$$

Substituting into (2.4) and using $n-m=\ell$ give

$$
\begin{aligned}
\Delta_{n} & \leq n+m-2 \int S_{n}^{2}(y) K_{n}\left(\mu_{n}, y, y\right) d v_{n}(y)+2(n-m) \\
& =2 n+\ell-2 \int S_{n}^{2}(y) K_{n}\left(\mu_{n}, y, y\right) d v_{n}(y) .
\end{aligned}
$$

Using the inequality $(x+y)^{2} \leq 2\left(x^{2}+y^{2}\right)$, we see that

$$
\begin{aligned}
n \Gamma_{n} & \leq 2 \Delta_{n}+2 \iint\left(K_{n}\left(\mu_{n}, x, y\right)-K_{m}\left(\mu_{n}, x, y\right)\right)^{2} S_{n}^{2}(x) S_{n}^{2}(y) d v_{n}(x) d v_{n}(y) \\
& \leq 2 \Delta_{n}+2 \iint\left(K_{n}\left(\mu_{n}, x, y\right)-K_{m}\left(\mu_{n}, x, y\right)\right)^{2} d \mu_{n}(x) d \mu_{n}(y) \\
& =2 \Delta_{n}+2(n-m)=2 \Delta_{n}+2 \ell .
\end{aligned}
$$

Now use (2.5).

Under the hypotheses of Theorem 1.1, we can estimate $\Gamma_{n}$ defined by (2.3).

Lemma 2.2. Assume the hypotheses of Theorem 1.1. Then

$$
\lim _{n \rightarrow \infty} \Gamma_{n}=0 \text {. }
$$

Proof. By our hypotheses,

$$
\frac{1}{n} K_{n}\left(\mu_{n}, x, x\right) v_{n}^{\prime}(x) S_{n}^{2}(x)=\frac{1}{n} K_{n}\left(\mu_{n}, x, x\right) \mu_{n}^{\prime}(x)\left[S_{n}^{2}(x) g_{n}(x)\right] \rightarrow \omega_{Q}^{\prime}
$$

in measure in $\mathcal{S}_{Q}$ as $n \rightarrow \infty$. Let $\varepsilon>0$ and

$$
\mathcal{E}_{n}=\left\{x \in \mathcal{S}_{Q}:\left|\frac{1}{n} K_{n}\left(\mu_{n}, x, x\right) v_{n}^{\prime}(x) S_{n}^{2}(x)-\omega_{Q}^{\prime}(x)\right|>\varepsilon\right\},
$$

so that meas $\left(\mathcal{E}_{n}\right) \rightarrow 0$ as $n \rightarrow \infty$. Then, as $n \rightarrow \infty$,

$$
\begin{aligned}
\frac{1}{n} \int & S_{n}^{2}(x) K_{n}\left(\mu_{n}, x, x\right) d v_{n}(x) \\
& \geq \int_{\mathcal{S}_{Q} \backslash \mathcal{E}_{n}}\left(\omega_{Q}^{\prime}(x)-\varepsilon\right) d x \\
& \rightarrow \int_{\mathcal{S}_{Q}}\left(\omega_{Q}^{\prime}(x)-\varepsilon\right) d x=1-\varepsilon
\end{aligned}
$$


Since $\varepsilon>0$ is arbitrary,

$$
\liminf _{n \rightarrow \infty} \frac{1}{n} \int S_{n}^{2}(x) K_{n}\left(\mu_{n}, x, x\right) d v_{n}(x) \geq 1
$$

because of integrability of $\omega_{Q}^{\prime}$. Together with our hypothesis (1.11) and (2.3), this gives (2.6).

\section{Potential Theoretic Estimates}

Next, we turn to some potential theoretic estimates. Given a positive measure $\rho$ with compact support in the plane, recall that [20]

$$
U^{\rho}(z)=\int \log \frac{1}{|z-t|} d \rho(t)
$$

denotes the associated potential. We need a general growth estimate.

LEMMA 3.1. Let $\rho$ be a measure with compact support in the real line and with total mass $\leq 1$. Let $\varepsilon>0$. There is a set $\mathcal{F} \subset \mathbb{R}$ with meas $(\mathcal{F})<\varepsilon$ such that, for $\xi \in \mathbb{R} \backslash \mathcal{F}$ and all $u \in \mathbb{C}$,

$$
U^{\rho}(\xi)-U^{\rho}(\xi+u) \leq C_{0}|u| / \varepsilon .
$$

Here $C_{0}$ is independent of $\rho, \xi, \varepsilon, u$.

Proof. This is essentially the same as that of Lemma 4.1 and Corollary 4.2 [18, pp. 232-233]. There estimates were made for the Green's function, but replacing the equilibrium measure by the measure $\rho$, we obtain essentially what we need. Nevertheless, we provide the details. Recall that the maximal function of the measure $\rho$ is

$$
\mathcal{M}[\rho](x)=\sup _{h>0} \frac{1}{2 h} \int_{x-h}^{x+h} d \rho .
$$

Moreover, $\mathcal{H}^{*}$ denotes the maximal Hilbert transform of $\rho$ defined by

$$
\mathcal{H}^{*}[\rho](x)=\sup _{\varepsilon>0}\left|\int_{|t-x| \geq \varepsilon} \frac{1}{t-x} d \rho(t)\right| .
$$

Step 1: A growth estimate. We first show that

$$
U^{\rho}(\xi)-U^{\rho}(\xi+u) \leq 26|u| \mathcal{M}[\rho](\xi)+|\operatorname{Re} u| \mathcal{H}^{*}[\rho](\xi) .
$$

Write $u=x+i y$. Now, for real $\xi$,

$$
\begin{aligned}
& U^{\rho}(\xi)-U^{\rho}(\xi+u) \\
& \quad=\frac{1}{2} \int \log \left[1+\frac{2 x}{\xi-t}+\frac{|u|^{2}}{(\xi-t)^{2}}\right] d \rho(t) .
\end{aligned}
$$

Let $\mathcal{S}_{1}$ denote the set of $t$ for which

$$
\left|\frac{2 x}{\xi-t}\right| \leq \frac{2|u|^{2}}{(\xi-t)^{2}} \Longleftrightarrow|\xi-t| \leq \frac{|u|^{2}}{|x|} .
$$


Let $\mathcal{S}_{2}$ denote the complementary range. In the case where $x=0$, of course, $\mathcal{S}_{2}$ is empty. Let us assume that $x \neq 0$; the case $x=0$ is easier. We see that

$$
\begin{aligned}
& \int_{\mathcal{S}_{1}} \log \left[1+\frac{2 x}{\xi-t}+\frac{|u|^{2}}{(\xi-t)^{2}}\right] d \rho(t) \\
& \leq \int_{|\xi-t| \leq \frac{|u|^{2}}{|x|}} \log \left[1+\frac{3|u|^{2}}{(\xi-t)^{2}}\right] d \rho(t) \\
& \leq \sum_{k=0}^{\infty} \int_{2^{-k-1} \frac{|u|^{2}}{|x|} \leq|\xi-t| \leq 2^{-k} \frac{|u|^{2}}{|x|}} \log \left[1+\frac{12 x^{2}}{|u|^{2}} 2^{2 k}\right] d \rho(t) \\
& \leq \sum_{k=0}^{\infty} \log \left[1+\frac{12 x^{2}}{|u|^{2}} 2^{2 k}\right] 2^{-k+1} \frac{|u|^{2}}{|x|} \mathcal{M}[\rho](\xi) \\
& \leq \frac{|u|^{2}}{|x|} \mathcal{M}[\rho](\xi) 4 \int_{0}^{\infty} \log \left[1+\frac{12 x^{2}}{|u|^{2} t^{2}}\right] d t \\
& =|u| \mathcal{M}[\rho](\xi) 8 \sqrt{3} \pi,
\end{aligned}
$$

cf. [12, p. 525, no. 4.222.1]. Next, in $\mathcal{S}_{2}$, we have $|\xi-t| \geq|u|^{2} /|x|$, so using the inequality $\log (1+t) \leq t, t \geq-1$, we obtain

$$
\begin{aligned}
\int_{\mathcal{S}_{2}} & \log \left[1+\frac{2 x}{\xi-t}+\frac{|u|^{2}}{(\xi-t)^{2}}\right] d \rho(t) \\
& \leq \int_{|\xi-t| \geq|u|^{2} /|x|}\left[\frac{2 x}{\xi-t}+\frac{|u|^{2}}{(\xi-t)^{2}}\right] d \rho(t) \\
& \leq 2|x|\left|\mathcal{H}^{*}[\rho](\xi)\right|+|u|^{2} \int_{|\xi-t| \geq|u|^{2} /|x|} \frac{1}{(\xi-t)^{2}} d \rho(t) .
\end{aligned}
$$

Here,

$$
\begin{aligned}
\int_{|\xi-t| \geq|u|^{2} /|x|} \frac{1}{(\xi-t)^{2}} d \rho(t) \\
\quad \leq \sum_{k=0}^{\infty} \int_{2^{k+1}|u|^{2} /|x| \geq|\xi-t| \geq 2^{k}|u|^{2} /|x|} \frac{1}{\left(2^{k}|u|^{2} /|x|\right)^{2}} d \rho(t) \\
\quad \leq \sum_{k=0}^{\infty} \frac{x^{2}}{|u|^{4}} 2^{-2 k} \cdot 2^{k+2} \frac{|u|^{2}}{|x|} \mathcal{M}[\rho](\xi) \\
\quad=\frac{|x|}{|u|^{2}} 8 \mathcal{M}[\rho](\xi) .
\end{aligned}
$$

Combining this with (3.3) and (3.4) gives

$$
\begin{aligned}
& U^{\rho}(\xi)-U^{\rho}(\xi+u) \\
& \quad \leq 4 \sqrt{3} \pi|u| \mathcal{M}[\rho](\xi)+|x|\left|\mathcal{H}^{*}[\rho](\xi)\right|+|x| 4 \mathcal{M}[\rho](\xi) .
\end{aligned}
$$

Estimating the constants gives (3.2). 
Step 2: Estimate the maximal function and Hilbert transform. Next, we use the fact that both the maximal function and the maximal Hilbert transform are weak type $(1,1)$. That is, [21, p. 137, Theorem 7.4],

$$
\operatorname{meas}\{\xi: \mathcal{M}[\rho](\xi)>\lambda\} \leq \frac{3}{\lambda} \int d \rho \leq \frac{3}{\lambda},
$$

and [3, p. 139], [11, p. $128 \mathrm{ff}$.

$$
\operatorname{meas}\left\{\xi: \mathcal{H}^{*}[\rho](\xi)>\lambda\right\} \leq \frac{C_{1}}{\lambda} \int d \rho \leq \frac{C_{1}}{\lambda} .
$$

Here $C_{1}$ is independent of $\rho$ and $\lambda$. Note that in these references the bound for $\mathcal{H}^{*}[\rho]$ is proved assuming that $\rho$ is absolutely continuous. However, it is true without this restriction. Indeed, John Garnett emailed to the authors about [11]: "The proof of Theorem 2.1 of Chapter III about the maximal conjugate function goes through with the $L_{1}$ function replaced by a finite measure. Also, on the line it is easy to estimate the difference between the truncated Hilbert transform of $|x|>$ $\varepsilon$ and the conjugate function at $i / \varepsilon$ by the ordinary maximal function". Choosing $\lambda=\frac{2}{\varepsilon} \max \left\{3, C_{1}\right\}$, we obtain a set $\mathcal{F}$ of measure $\leq \varepsilon$ such that, for $\xi \in \mathbb{R} \backslash \mathcal{F}$ and all complex $u,(3.1)$ holds with a constant $C_{0}$ independent of $\rho, \xi, u, \varepsilon$.

Lemma 3.2. Let $\Sigma$ be a closed set, let $Q: \Sigma \rightarrow[0, \infty)$ be such that $e^{-Q}$ is admissible, and let $\omega_{Q}$ denote the equilibrium measure for $e^{-Q}$, with support $\mathcal{S}_{Q}$. Let $L$ be a compact subset of $\Sigma$, and assume that $L$ is the closure of its (nonempty) interior. Let $n>\ell \geq 1$. Let $S_{n}$ be a polynomial of degree $\ell$ that has no zeros on $\Sigma$. Let $\varepsilon>0$, and $R: \mathbb{C} \rightarrow \mathbb{C}$ be a function such that $\log |R|$ is subharmonic in $\mathbb{C}$. Assume also that

$$
\lim _{|z| \rightarrow \infty}(\log |R(z)|-(n+\ell) \log |z|)
$$

exists and is finite and that

$$
|R(x)| e^{-n Q(x)} /\left|S_{n}(x)\right| \leq 1 \quad \text { for } x \in L .
$$

Then there exists a set $\mathcal{F}_{n} \subset \mathbb{R}$ with meas $\left(\mathcal{F}_{n}\right)<\varepsilon$ such that, for $\xi \in\left(L \cap \mathcal{S}_{Q}\right) \backslash \mathcal{F}_{n}$ and all $u \in \mathbb{C}$,

$$
|R(\xi+u)| e^{-n Q(\xi)} /\left|S_{n}(\xi)\right| \leq e^{C_{0} n|u| / \varepsilon}, \quad u \in \mathbb{C} .
$$

The set $\mathcal{F}_{n}$ depends on $Q, \varepsilon, n, \ell, S_{n}$ but not on the particular $R$. Also, $C_{0}$ is independent of $Q, R, n, \xi, \varepsilon, u$.

Proof. Recall that

$$
U^{\omega_{Q}}+Q=F_{Q} \quad \text { q.e. in } \mathcal{S}_{Q}
$$

Now balayage that part of $\omega_{Q}$ with support in $\mathcal{S}_{Q} \backslash L^{o}$ onto $L$. More precisely, we apply Theorem II.4.7 in [22, p. 116] with the domain $G=\mathbb{C} \backslash L$ giving the balayage measure

$$
v=\widehat{\omega_{Q \mid G}} \text {. }
$$

It has the property that, for some constant $C_{0}$,

$$
U^{v}(x)=U^{\omega} Q \mid G(x)+C_{0}, \quad \text { q.e. } x \in L .
$$


If we let

$$
\tilde{\omega}_{Q}=\omega_{Q \mid L}+v
$$

(the sum of $v$ and the restriction of $\omega_{Q}$ to $L$ ), then for q.e. $x \in L \cap \mathcal{S}_{Q}$,

$$
U^{\tilde{\omega}} Q(x)=U^{\omega} Q(x)+C_{0}=-Q(x)+F_{Q}+C_{0}
$$

(recall (1.6)). Note that $\tilde{\omega}_{Q}$ has support in $L$. Next, we may assume that $S_{n}$ is monic (a constant can be absorbed into $R$ ). Then we can write

$$
S_{n}(x)=\prod_{j=1}^{\ell}\left(x-b_{j}\right),
$$

and if

$$
\rho=\sum_{j=1}^{\ell} \delta_{b_{j}}
$$

is a measure with compact support in $\mathbb{C} \backslash \Sigma$, then we have

$$
-\log \left|S_{n}(z)\right|=U^{\rho}(z) .
$$

Let $\tilde{\rho}$ denote the balayage measure of $\rho$ onto $L \cap \mathcal{S}_{Q}$, so that by [22, Theorem II.4.7, p. 116] it has total mass $\ell$, support in $L \cap \mathcal{S}_{Q}$, and for some constant $C_{1}$,

$$
U^{\tilde{\rho}}(x)=U^{\rho}(x)+C_{1} \quad \text { q.e. in } L \cap \mathcal{S}_{Q} .
$$

Let

$$
C_{2}=n\left(F_{Q}+C_{0}\right)+C_{1} .
$$

Then for q.e. $x \in L \cap \mathcal{S}_{Q}$, we have from (3.8) and (3.9)

$$
e^{n U^{\tilde{\omega}} Q(x)+U^{\tilde{\rho}}(x)-C_{2}}=e^{-n Q(x)} /\left|S_{n}(x)\right|,
$$

so we can recast (3.6) as

$$
|R(x)| e^{n U^{\tilde{\omega}} Q(x)+U^{\tilde{\rho}}(x)-C_{2}} \leq 1 \quad \text { for q.e. } x \in L \cap \mathcal{S}_{Q} .
$$

Moreover, since $\tilde{\omega}_{Q}$ and $\tilde{\rho}$ have supports in $L \cap \mathcal{S}_{Q}$, their potentials are harmonic outside this set, so $\log |R(z)|+n U^{\tilde{\omega}} Q(z)+U^{\tilde{\rho}}(z)-C_{2}$ is subharmonic in $\mathbb{C} \backslash(L \cap$ $\mathcal{S}_{Q}$ ), with a finite limit at $\infty$ in view of (3.5). By the maximum principle for subharmonic functions, we have

$$
|R(z)| e^{n U^{\tilde{\omega}} Q}(z)+U^{\tilde{\rho}}(z)-C_{2} \leq 1, \quad z \in \mathbb{C} .
$$

Now let

$$
\omega:=\frac{1}{n+\ell}\left[n \tilde{\omega}_{Q}+\tilde{\rho}\right],
$$

which is a measure of total mass 1 with support in $L \cap \mathcal{S}_{Q}$. From (3.11) we have

$$
|R(z)| e^{(n+\ell) U^{\omega}(z)-C_{2}} \leq 1, \quad z \in \mathbb{C} .
$$

Then for $\xi \in \mathbb{R}$ and complex $u$, we have

$$
|R(\xi+u)| e^{(n+\ell) U^{\omega}(\xi)-C_{2}} \leq e^{(n+\ell)\left[U^{\omega}(\xi)-U^{\omega}(\xi+u)\right]},
$$


and now we can apply Lemma 3.1. Note that the set $\mathcal{F}_{n}$ in Lemma 3.1 does not depend on the particular $R$ since $\omega$ does not depend on $R$. Finally, by (3.10), in $L \cap \mathcal{S}_{Q}$, we have

$$
e^{(n+\ell) U^{\omega}(\xi)-C_{2}}=e^{-n Q(\xi)} /\left|S_{n}(\xi)\right| .
$$

\section{Pointwise Estimates and Proof of Theorem 1.1}

Throughout, we assume the hypotheses of Theorem 1.1 and the notation of the previous sections. In particular, $\Gamma_{n}$ is defined by (2.3). We begin with the following:

Lemma 4.1. Let $\varepsilon>0$. For $n \geq n_{0}(\varepsilon)$, there exists a set $\mathcal{G}_{n}$ of measure $\leq \Gamma_{n}^{1 / 2}+\varepsilon$ such that, for $\xi \in \mathcal{S}_{Q} \backslash \mathcal{G}_{n}$ and for all $u, z \in \mathbb{C}$, we have

$$
\begin{gathered}
\frac{1}{n}\left|K_{n}\left(v_{n}, \xi+u, z\right)-S_{n}(\xi+u) S_{n}(z) K_{n}\left(\mu_{n}, \xi+u, z\right)\right| v_{n}^{\prime}(\xi) \\
\leq 4\left[\frac{1}{n} K_{n+\ell}\left(v_{n}, z, \bar{z}\right) v_{n}^{\prime}(\xi)\right]^{1 / 2} \Gamma_{n}^{1 / 4} e^{C_{0} n|u| / \varepsilon} .
\end{gathered}
$$

Proof. Step 1: An integral estimate and exceptional set. Let $\mathcal{E}_{n}$ denote the set of $x \in \Sigma$ for which

$$
\frac{1}{n} \int\left(K_{n}\left(v_{n}, x, y\right)-S_{n}(x) S_{n}(y) K_{n}\left(\mu_{n}, x, y\right)\right)^{2} d v_{n}(y) v_{n}^{\prime}(x)>\sqrt{\Gamma_{n}} .
$$

Then

$$
\begin{aligned}
& \operatorname{meas}\left(\mathcal{E}_{n}\right) \sqrt{\Gamma_{n}} \\
& \quad \frac{1}{n} \iint\left(K_{n}\left(v_{n}, x, y\right)-S_{n}(x) S_{n}(y) K_{n}\left(\mu_{n}, x, y\right)\right)^{2} d v_{n}(y) d v_{n}(x)=\Gamma_{n} \\
& \quad \Rightarrow \operatorname{meas}\left(\mathcal{E}_{n}\right) \leq \sqrt{\Gamma_{n}} .
\end{aligned}
$$

Step 2: A Christoffel function estimate.

We use the inequality

$$
|P(z)|^{2} \leq K_{n+\ell}\left(v_{n}, z, \bar{z}\right) \int|P|^{2} d v_{n},
$$

valid for all polynomials $P$ of degree $<n+\ell$ and all $z \in \mathbb{C}$, applied to

$$
P(z)=\frac{1}{n}\left(K_{n}\left(v_{n}, x, z\right)-S_{n}(x) S_{n}(z) K_{n}\left(\mu_{n}, x, z\right)\right)
$$

with $x \in \Sigma \backslash \mathcal{E}_{n}$ fixed. We then obtain from Step 1, for $x \in \Sigma \backslash \mathcal{E}_{n}$ and all $z \in \mathbb{C}$,

$$
\begin{aligned}
& \frac{1}{n^{2}}\left|K_{n}\left(v_{n}, x, z\right)-S_{n}(x) S_{n}(z) K_{n}\left(\mu_{n}, x, z\right)\right|^{2} v_{n}^{\prime}(x) \\
& \quad=|P(z)|^{2} v_{n}^{\prime}(x) \\
& \quad \leq K_{n+\ell}\left(v_{n}, z, \bar{z}\right)\left(\int|P|^{2} d v_{n}\right) v_{n}^{\prime}(x) \leq \frac{1}{n} K_{n+\ell}\left(v_{n}, z, \bar{z}\right) \Gamma_{n}^{1 / 2} .
\end{aligned}
$$


Now take square roots: for $x \in \Sigma \backslash \mathcal{E}_{n}$ and all $z \in \mathbb{C}$,

$$
\begin{aligned}
& \frac{1}{n}\left|K_{n}\left(v_{n}, x, z\right)-S_{n}(x) S_{n}(z) K_{n}\left(\mu_{n}, x, z\right)\right| v_{n}^{\prime}(x)^{1 / 2} \\
& \leq\left(\frac{1}{n} K_{n+\ell}\left(v_{n}, z, \bar{z}\right)\right)^{1 / 2} \Gamma_{n}^{1 / 4} .
\end{aligned}
$$

Step 3: Fix $z$ and apply Lemma 3.2.

Fix $n \geq 1$, fix $z \in \mathbb{C}$ with $S_{n}(z) p_{n-1}(z) \neq 0$, let $L_{n}=\mathcal{S}_{Q} \backslash \mathcal{E}_{n}$ (which is independent of $z$ ), and denote

$$
\begin{aligned}
A_{n} & =\left[\frac{1}{n} K_{n+\ell}\left(v_{n}, z, \bar{z}\right)\right]^{1 / 2} \Gamma_{n}^{1 / 4} ; \\
R(t) & =\frac{1}{n A_{n}}\left(K_{n}\left(v_{n}, t, z\right)-S_{n}(t) S_{n}(z) K_{n}\left(\mu_{n}, t, z\right)\right) .
\end{aligned}
$$

Then $\log |R(t)|$ is subharmonic in the plane, and (assuming that $S_{n}$ has full degree $\ell \geq 1$, as we may) $\log |R(t)|-(n+\ell-1) \log |t|$ has a finite limit as $|t| \rightarrow \infty$ since we assumed that $\left(S_{n} p_{n-1}\right)(z) \neq 0$. Moreover, for $x \in L_{n}$, we can recast (4.3) as

$$
|R(x)| h(x)^{1 / 2} e^{-n Q(x)} g_{n}(x)^{1 / 2} \leq 1 .
$$

(Recall (1.8) and (1.10).) Since $h$ is bounded above and below by positive constants in $\Sigma$ and does not depend on $n$, we can simply assume that $h \equiv 1$. Next, by our hypothesis that $g_{n} S_{n}^{2} \rightarrow 1$ in measure in $\mathcal{S}_{Q}$, for large enough $n$, we may replace $g_{n}^{1 / 2}$ in this last inequality by $\frac{1}{2}\left|S_{n}\right|^{-1}$ except on a set of small measure. Absorbing that set of small measure into $\mathcal{E}_{n}$ (perhaps by replacing $\varepsilon$ by $\varepsilon / 2$ ), we obtain, for large enough $n$,

$$
\frac{1}{2}|R(x)| e^{-n Q(x)}\left|S_{n}(x)\right|^{-1} \leq 1, \quad x \in L_{n} .
$$

Since the left-hand side is continuous, we may assume that $L_{n}$ is closed and (by removing isolated points) is even the closure of its interior. Moreover, the new $L_{n}$ is still independent of $z$ above. Then by Lemma 3.2 there exists a set $\mathcal{F}_{n} \subset \mathbb{R}$ with meas $\left(\mathcal{F}_{n}\right)<\varepsilon$ such that, for $\xi \in L_{n} \backslash \mathcal{F}_{n}=\mathcal{S}_{Q} \backslash\left(\mathcal{E}_{n} \cup \mathcal{F}_{n}\right)$ and all $u \in \mathbb{C}$,

$$
\begin{aligned}
& \frac{1}{2 n A_{n}}\left|K_{n}\left(v_{n}, \xi+u, z\right)-S_{n}(\xi+u) S_{n}(z) K_{n}\left(\mu_{n}, \xi+u, z\right)\right| e^{-n Q(\xi)} /\left|S_{n}(\xi)\right| \\
& \quad \leq e^{C_{0} n|u| / \varepsilon} .
\end{aligned}
$$

Note that the set $\mathcal{F}_{n}$ did not depend on the particular $R$, so it works for all complex $z$. We may replace $e^{-n Q(\xi)} /\left|S_{n}(\xi)\right|$ by $v_{n}^{\prime}(\xi)^{1 / 2}$ in this last inequality, at the cost of adding a set of small measure to $\mathcal{E}_{n} \cup \mathcal{F}_{n}$. Finally, we set $\mathcal{G}_{n}=\mathcal{E}_{n} \cup \mathcal{F}_{n}$, multiply by $v_{n}^{\prime}(\xi)^{1 / 2}$, and take into account the definition of $A_{n}$ to obtain the result, at least when $\left(S_{n} p_{n-1}\right)(z) \neq 0$. Since this last condition holds except at finitely many $z$, we can use continuity in $z$ to deduce (4.1) for such $z$ also.

Next, we estimate $K_{n+\ell}$. We shall assume that in $\mu_{n}^{\prime}, h \equiv 1$. The general case involves trivial modifications. 
LEMmA 4.2. Let $\varepsilon>0$. There exists a set $\mathcal{H}_{n}$ of measure at most $2 \varepsilon$ such that, for $\xi \in \mathcal{S}_{Q} \backslash \mathcal{H}_{n}$ and all complex $v$,

$$
\frac{1}{n}\left|K_{n+\ell}\left(v_{n}, \xi+v, \xi+\bar{v}\right)\right| v_{n}^{\prime}(\xi) \leq \frac{4}{\varepsilon} e^{n C_{1}|v| / \varepsilon},
$$

where $C_{1}$ is independent of $n, \xi, v, \varepsilon$.

Proof. We shall abbreviate $p_{k}\left(v_{n}, z\right)$ as $p_{k}(z)$. First, we note that, for $n \geq 1$,

$$
\log K_{n}\left(v_{n}, z, \bar{z}\right)=\log \left(\sum_{k=0}^{n-1}\left|p_{k}(z)\right|^{2}\right)
$$

is subharmonic in the plane. Indeed, if we fix $z$ and $r>0$, we can choose unimodular constants $\left\{\alpha_{k}\right\}$ such that

$$
\begin{aligned}
\log K_{n}\left(v_{n}, z, \bar{z}\right) & =\log \left|\sum_{k=0}^{n-1} \alpha_{k} p_{k}(z)^{2}\right| \\
& \leq \frac{1}{2 \pi} \int_{-\pi}^{\pi} \log \left|\sum_{k=0}^{n-1} \alpha_{k} p_{k}\left(z+r e^{i \theta}\right)^{2}\right| d \theta \\
& \leq \frac{1}{2 \pi} \int_{-\pi}^{\pi} \log \left(\sum_{k=0}^{n-1}\left|p_{k}\left(z+r e^{i \theta}\right)\right|^{2}\right) d \theta \\
& =\frac{1}{2 \pi} \int_{-\pi}^{\pi} \log K_{n}\left(v_{n}, z+r e^{i \theta}, \overline{z+r e^{i \theta}}\right) d \theta .
\end{aligned}
$$

In the second last line, we used the subharmonicity of absolute values of analytic functions. Moreover, we see that, as $|z| \rightarrow \infty$,

$$
\log K_{n}\left(v_{n}, z, \bar{z}\right)=(2 n-2) \log |z|+2 \log \gamma_{n-1}+o(1) .
$$

Now, for small $\varepsilon>0$, let

$$
\mathcal{I}_{n}=\left\{t \in \mathcal{S}_{Q}: \frac{1}{n} K_{n+\ell}\left(v_{n}, t, t\right) v_{n}^{\prime}(t) \geq \frac{2}{\varepsilon}\right\} .
$$

Observe that

$$
\operatorname{meas}\left(\mathcal{I}_{n}\right) \frac{2}{\varepsilon} \leq \int \frac{1}{n} K_{n+\ell}\left(v_{n}, t, t\right) d v_{n}(t)<2
$$

since $n>\ell$, so

$$
\operatorname{meas}\left(\mathcal{I}_{n}\right) \leq \varepsilon
$$

Since $S_{n}^{2} g_{n} \rightarrow 1$ in measure, we may absorb a set of small measure into $\mathcal{I}_{n}$ and obtain

$$
\frac{1}{2 n} K_{n+\ell}\left(v_{n}, t, t\right) e^{-2 n Q(t)} / S_{n}^{2}(t) \leq \frac{2}{\varepsilon}, \quad t \in \mathcal{S}_{Q} \backslash \mathcal{I}_{n} .
$$

We now let $L_{n}=\mathcal{S}_{Q} \backslash \mathcal{I}_{n}$. Since the left-hand side in this last inequality is continuous, we may assume that $L_{n}$ is closed and (by removing isolated points) is also the closure of its interior. Next, by Lemma 3.2 with $R(t)=K_{n+\ell}\left(v_{n}, t, \bar{t}\right)$, with 
$L$ taken as $L_{n}, Q$ replaced by $2 Q$, and $S_{n}$ by $S_{n}^{2}$, there is a set $\mathcal{J}_{n}$ of measure at most $\varepsilon$ such that, for $\xi \in L_{n} \backslash \mathcal{J}_{n}=\mathcal{S}_{Q} \backslash\left(\mathcal{I}_{n} \cup \mathcal{J}_{n}\right)$ and all complex $v$,

$$
\frac{\varepsilon}{4 n} K_{n+\ell}\left(v_{n}, \xi+v, \overline{\xi+v}\right) e^{-2 n Q(\xi)} / S_{n}^{2}(\xi) \leq e^{n C_{1}|v| / \varepsilon}
$$

Here $C_{1}$ is independent of $n, v, \varepsilon, \xi$. Setting $\mathcal{H}_{n}=\mathcal{I}_{n} \cup \mathcal{J}_{n}$, we see that meas $\left(\mathcal{H}_{n}\right)<2 \varepsilon$, and for $\xi \in \mathcal{S}_{Q} \backslash \mathcal{H}_{n}$ and all complex $v$, we have (4.4), except that $\nu_{n}^{\prime}$ is replaced by $e^{-2 n Q(\xi)} / S_{n}^{2}(\xi)$. Again, we can add a small set to $\mathcal{H}_{n}$ and replace $e^{-2 n Q(\xi)} / S_{n}^{2}(\xi)$ by $\nu_{n}^{\prime}(\xi)$.

Now we put it all together:

Proof of Theorem 1.1. We apply Lemmas 4.1 and 4.2: let $\mathcal{E}_{n}=\mathcal{G}_{n} \cup \mathcal{H}_{n}$, so that $\operatorname{meas}\left(\mathcal{E}_{n}\right)<3 \varepsilon+2 \Gamma_{n}^{1 / 2}$ and, for $\xi \in \mathcal{S}_{Q} \backslash \mathcal{E}_{n}$ and all $u, v \in \mathbb{C}$,

$$
\begin{aligned}
& \frac{1}{n}\left|K_{n}\left(v_{n}, \xi+u, \xi+v\right)-S_{n}(\xi+u) S_{n}(\xi+v) K_{n}\left(\mu_{n}, \xi+u, \xi+v\right)\right| v_{n}^{\prime}(\xi) \\
& \leq \frac{8}{\sqrt{\varepsilon}} \Gamma_{n}^{1 / 4} e^{C_{0} n(|u|+|v|) / \varepsilon} .
\end{aligned}
$$

Finally, we know that (2.6) holds, so we can just choose $u=a / n, v=b / n$, and replace $\varepsilon$ by $\varepsilon / 4$.

For future use, we record this last inequality as the following:

Theorem 4.3. Assume the hypotheses of Theorem 1.1. Let $\varepsilon>0$. Then, for $n \geq 1$, there exists a set $\mathcal{E}_{n}$ of measure $\leq \varepsilon$ such that, for $\xi \in S_{Q} \backslash \mathcal{E}_{n}$ and all complex $a, b$,

$$
\begin{aligned}
\frac{1}{n} \mid K_{n} & \left(v_{n}, \xi+\frac{a}{n}, \xi+\frac{b}{n}\right) \\
& \quad-S_{n}\left(\xi+\frac{a}{n}\right) S_{n}\left(\xi+\frac{b}{n}\right) K_{n}\left(\mu_{n}, \xi+\frac{a}{n}, \xi+\frac{b}{n}\right) \mid v_{n}^{\prime}(\xi) \\
\leq & \frac{8}{\sqrt{\varepsilon}} \Gamma_{n}^{1 / 4} e^{C_{0}(|a|+|b|) / \varepsilon} .
\end{aligned}
$$

Here $C_{0}$ is independent of $n, \xi, a, b, \varepsilon$. We may replace $v_{n}^{\prime}(\xi)$ by $\mu_{n}^{\prime}(\xi) / S_{n}^{2}(\xi)$ in this last left-hand side.

\section{Proof of Theorem 1.2}

Our main task is to find approximating polynomials $\left\{S_{n}\right\}$ satisfying hypotheses (1.11)-(1.13) of Theorem 1.1. We begin with the following:

LEMMA 5.1. Let $\left\{\delta_{n}\right\} \subset(0, \infty)$ with

$$
\lim _{n \rightarrow \infty} \delta_{n}=0 .
$$

Let $r>0$. There exist nonnegative polynomials $\left\{P_{n}\right\}$ with

$$
\operatorname{deg}\left(P_{n}\right)=o(n), \quad n \rightarrow \infty,
$$


and, as $n \rightarrow \infty$, uniformly in compact subsets of $(-r, r) \backslash\{0\}$,

$$
|x|^{n \delta_{n}} P_{n}(x) \rightarrow 1
$$

while for $n \geq 1$,

$$
\sup _{x \in[-r, r]}|x|^{n \delta_{n}} P_{n}(x) \leq 1 .
$$

Proof. First, note that by dilating the variable we may assume that $r=1$. Next, it suffices to find nonnegative polynomials $\left\{P_{n}\right\}$ of degree $o(n)$ satisfying (5.2) and also, as $n \rightarrow \infty$,

$$
x^{n \delta_{n}} P_{n}(x) \rightarrow 1 \quad \text { uniformly in compact subsets of }(0,1),
$$

whereas, for $n \geq 1$,

$$
0 \leq x^{n \delta_{n}} P_{n}(x) \leq 1 \quad \text { in }[0,1] .
$$

Indeed, we can always replace $P_{n}(x)$ satisfying these last two conditions by $P_{n}\left(x^{2}\right)$, after also replacing $\delta_{n}$ by $\delta_{n} / 2$.

Next, we recall classical results from the theory of incomplete polynomials. Let $\theta \in\left(0, \frac{1}{4}\right)$, and $f_{\theta}:[0,1] \rightarrow[0,1]$ be continuous with $f_{\theta}=0$ in $\left[0, \theta^{2}\right] \cup\{1\}$. Then by [22, p. 283] there exist polynomials $R_{m, \theta}$ of degree $\leq m$ such that

$$
\lim _{m \rightarrow \infty} x^{m \theta /(1-\theta)} R_{m, \theta}(x)=f_{\theta}(x)
$$

uniformly in [0,1]. We may also assume that $R_{m, \theta} \geq 0$ in [0,1]. (If not consider $R_{m, \theta}^{2}$ after modifying the value of $\theta$ ). By dividing by $R_{m, \theta}$ by $1+\eta_{m}$, where $\eta_{m}$ decreases to 0 sufficiently slowly, we can also assume that

$$
0 \leq x^{m \theta /(1-\theta)} R_{m, \theta}(x)<1 \quad \text { in }[0,1] .
$$

We shall choose our $f_{\theta}$ so that $0 \leq f_{\theta} \leq 1$ in $[0,1]$ and $f_{\theta}=1$ in $[\theta, 1-\theta]$.

Next, fix $\varepsilon \in\left(0, \frac{1}{2}\right)$ and set $\theta=\frac{\varepsilon}{2}$. For large enough $n$, write

$$
\frac{1-\theta}{\theta} \delta_{n} n=m_{n}+\Delta_{n}
$$

where $m_{n}$ is a positive integer, and $\Delta_{n} \in[0,1)$. We shall set, for some suitable polynomial $V_{n}$,

$$
P_{n}(x)=R_{m_{n}, \theta}(x) V_{n}(x),
$$

so that

$$
x^{\delta_{n} n} P_{n}(x)=\left(x^{m_{n} \theta /(1-\theta)} R_{m_{n}, \theta}(x)\right)\left(x^{\frac{\theta}{1-\theta} \Delta_{n}} V_{n}(x)\right) .
$$

Assume now that $\operatorname{deg}\left(V_{n}\right) \leq \log n$ and

$$
0 \leq x^{\frac{\theta}{1-\theta} \Delta_{n}} V_{n}(x) \leq 1 \quad \text { in }[0,1],
$$

while uniformly in compact subsets of $(0,1]$,

$$
\lim _{n \rightarrow \infty} x^{\frac{\theta}{1-\theta} \Delta_{n}} V_{n}(x)=1 .
$$

Then $P_{n}$ has degree $\leq m_{n}+\log n \leq \frac{1-\theta}{\theta} \delta_{n} n+\log n$, and by (5.8) and (5.9)

$$
0 \leq x^{\delta_{n} n} P_{n}(x) \leq 1 \text {, }
$$


while uniformly in $\left[\frac{\varepsilon}{2}, 1-\frac{\varepsilon}{2}\right]$, by $(5.7),(5.10)$, and our choice of $f_{\theta}$,

$$
\lim _{n \rightarrow \infty} x^{\delta_{n} n} P_{n}(x)=1 \text {. }
$$

(Recall that $f_{\theta}=1$ in $\left[\frac{\varepsilon}{2}, 1-\frac{\varepsilon}{2}\right]=[\theta, 1-\theta]$.) The conclusion of the lemma then follows by choosing a sufficiently slowly decreasing sequence of values for $\varepsilon$ and corresponding $\left\{P_{n}\right\}$ (with "long" subsequences of $\left\{P_{n}\right\}$ arising from each of the values of $\varepsilon$ ).

It remains to prove the existence of $\left\{V_{n}\right\}$ satisfying (5.9) and (5.10). Let

$$
A_{\ell}(t)=-\sum_{j=1}^{\ell} \frac{t^{j}}{j}
$$

denote the $\ell$ th partial sum of the Maclaurin series of $\log (1-t)$, so that

$$
A_{\ell}(t) \geq \log (1-t), \quad t \in[0,1),
$$

and

$$
\sup _{t \in[0,1]}\left|A_{\ell}(t)\right| \leq 1+\log \ell .
$$

Also, let $B_{\ell}$ denote the $\ell$ th partial sum of the Maclaurin series of $\exp (-t)$, so that, by straightforward calculations,

$$
\sup _{|t| \leq \ell / 10}\left|B_{\ell}(t) \exp (t)-1\right|=o(1) \quad \text { as } \ell \rightarrow \infty .
$$

We let $\ell=[\sqrt{\log n}]$ (where $[x]$ denotes the greatest integer $\leq x)$ and

$$
V_{n}(x)=B_{[\sqrt{\log n}]}\left(-\frac{\theta}{1-\theta} \Delta_{n} A_{[\sqrt{\log n}]}(1-x)\right),
$$

so that, in view of (5.11)-(5.13), uniformly for $x \in[0,1]$,

$$
\begin{aligned}
0 & \leq x^{\frac{\theta}{1-\theta} \Delta_{n}} V_{n}(x) \\
& =(1+o(1)) \exp \left(\frac{\theta}{1-\theta} \Delta_{n}\left\{\log (1-(1-x))-A_{[\sqrt{\log n}]}(1-x)\right\}\right) \\
& \leq 1+o(1) .
\end{aligned}
$$

Moreover, the desired uniform convergence in compact subsets of $(0,1]$ in $(5.10)$ follows from the convergence of the partial sums $\left\{A_{\ell}\right\}$ and $\left\{B_{\ell}\right\}$. The factor $1+$ $o$ (1) may be replaced by 1 by dividing by $1+\eta_{n}$ for some slowly decreasing sequence $\left\{\eta_{n}\right\}$ with limit 0 .

Next, we handle the factors $e^{\tau_{n} \varphi_{n}}$.

Lemma 5.2. Let $\alpha,\left\{\tau_{n}\right\}$, and $\left\{\varphi_{n}\right\}$ be as in Theorem 1.2. Then there exist polynomials $\left\{R_{n}\right\}$ with

$$
\begin{gathered}
\operatorname{deg} R_{n}=o(n), \quad n \rightarrow \infty \\
0 \leq R_{n}(x) e^{\tau_{n} \varphi_{n}(x)} \leq 1, \quad x \in \Sigma \\
\lim _{n \rightarrow \infty} R_{n}(x) e^{\tau_{n} \varphi_{n}(x)}=1 \quad \text { uniformly in } \Sigma .
\end{gathered}
$$


Proof. For the purposes of this lemma, we can assume that $\Sigma$ is a single closed interval since we can extend the domain of definition of the functions $\left\{\varphi_{n}\right\}$ without increasing the sup norms of $\varphi_{n}$ and their derivatives or Lipschitz norms. Let

$$
\ell_{n}=\left[n^{\frac{1}{1+\alpha}}\right], \quad n \geq 1,
$$

so that by $(1.18)$

$$
\lim _{n \rightarrow \infty} \tau_{n} / \ell_{n}^{\alpha}=0
$$

By Jackson's theorem [7, Theorem 6.2, p. 219] and our hypothesis of uniform smoothness of order $\alpha$ we can find a polynomial $A_{n}$ of degree $\leq \ell_{n}$ such that

$$
\left\|\varphi_{n}-A_{n}\right\|_{L_{\infty}(\Sigma)} \leq C / \ell_{n}^{\alpha}
$$

Here $C$ is independent of $n$. Then

$$
\left\|\tau_{n} \varphi_{n}-\tau_{n} A_{n}\right\|_{L_{\infty}(\Sigma)} \leq C \tau_{n} / \ell_{n}^{\alpha} \rightarrow 0, \quad n \rightarrow \infty .
$$

By adding a sequence of positive numbers with limit 0 we may assume that

$$
\tau_{n} \varphi_{n} \leq \tau_{n} A_{n} \quad \text { in } \Sigma \text {. }
$$

Next, let $B_{n}(t)$ denote the $n$th partial sum of the Maclaurin series of $e^{t}$. We use (5.13) and choose

$$
R_{n}(x)=B_{10\left[\tau_{n}\right]}\left(-\tau_{n} A_{n}(x)\right) .
$$

Note that this has degree at most $10\left[\tau_{n}\right] \ell_{n}=o\left(\ell_{n}^{1+\alpha}\right)=o(n)$ by (5.17) and (5.18). Also,

$$
\left\|\tau_{n} A_{n}\right\|_{L_{\infty}(\Sigma)} \leq \tau_{n}\left(1+O\left(1 / \ell_{n}^{\alpha}\right)\right)=\tau_{n}+o(1),
$$

so (5.13), followed by (5.20), shows that, uniformly for $x \in \Sigma$,

$$
R_{n}(x) e^{\tau_{n} \varphi_{n}(x)}=\exp \left(\tau_{n}\left(\varphi_{n}(x)-A_{n}(x)\right)\right)(1+o(1)) \leq 1+o(1),
$$

and by (5.19) we also have, uniformly for $x \in \Sigma$,

$$
R_{n}(x) e^{\tau_{n} \varphi_{n}(x)}=\exp \left(\tau_{n} O\left(1 / \ell_{n}^{\alpha}\right)\right)=1+o(1) .
$$

Now multiply $R_{n}$ by a sequence that is $1+o(1)$ to ensure inequality (5.15).

We need one more lemma on local growth of $v_{n}^{\prime}$.

Lemma 5.3. Let $R, \varepsilon>0$. There exist $C>1$ and, for $n \geq 1$, a set $\mathcal{E}_{n}$ of measure $<\varepsilon$ such that

$$
C^{-1} \leq \sup _{\xi \in \mathcal{S}_{Q} \backslash \mathcal{E}_{n},|a| \leq R} \frac{v_{n}^{\prime}(\xi)}{v_{n}^{\prime}\left(\xi+\frac{a}{n \omega_{Q}^{\prime}(\xi)}\right)} \leq C .
$$

Proof. In view of the form (1.15) of $v_{n}^{\prime}$, it suffices to show the following three estimates:

$$
\begin{aligned}
& C^{-1} \leq \sup _{\xi \in \mathcal{S}_{Q} \backslash \mathcal{E}_{n, 1},|a| \leq R} e^{\tau_{n}\left[\varphi_{n}(\xi)-\varphi_{n}\left(\xi+\frac{a}{n \omega^{\prime}(\xi)}\right)\right]} \leq C, \\
& C^{-1} \leq \sup _{\xi \in \mathcal{S}_{Q} \backslash \mathcal{E}_{n, 2},|a| \leq R}\left|\frac{\xi-b_{n}}{\xi-b_{n}+\frac{a}{n \omega_{Q}^{\prime}(\xi)}}\right|^{n \beta_{n}} \leq C,
\end{aligned}
$$




$$
C^{-1} \leq \sup _{\xi \in \mathcal{S}_{Q} \backslash \mathcal{E}_{n, 3},|a| \leq R} e^{2 n\left[Q(\xi)-Q\left(\xi+\frac{a}{n \omega^{\prime}(\xi)}\right)\right]} \leq C,
$$

where $\mathcal{E}_{n, j}, j=1,2,3$, are sets of small measure, and where $\lim _{n \rightarrow \infty} \beta_{n}=0$, while $\left\{b_{n}\right\}$ is a sequence of real numbers. The proof of (5.22) is easy and follows from the mean value theorem or from our assumed Lipschitz condition (if $\alpha \leq 1$ ). Note that since $\Sigma$ consists of finitely many intervals, $\xi$ and $\xi+\frac{a}{n \omega_{Q}^{\prime}(\xi)}$ belong to the same interval for large enough $n$ and for $\xi$ outside a set $\mathcal{E}_{n, 1}$ of small measure (outside which $\omega_{Q}^{\prime}$ is bounded above and below by specified constants). Then, recalling (1.18), we have

$$
\begin{gathered}
\sup _{\xi \in \mathcal{S}_{Q} \backslash \mathcal{E}_{n, 1},|a| \leq R} \tau_{n}\left|\varphi_{n}(\xi)-\varphi_{n}\left(\xi+\frac{a}{n \omega_{Q}^{\prime}(\xi)}\right)\right| \\
\leq \tau_{n} C\left(\frac{R}{n \inf _{\xi \in \mathcal{S}_{Q} \backslash \mathcal{E}_{n, 1}} \omega_{Q}^{\prime}(\xi)}\right)^{\min \{1, \alpha\}} \\
\quad=o\left(n^{\frac{\alpha}{1+\alpha}-\min \{1, \alpha\}}\right) \rightarrow 0, \quad n \rightarrow \infty .
\end{gathered}
$$

So we have $(5.22)$.

Next, for $|a| \leq R$,

$$
\begin{aligned}
& \left|\frac{\xi-b_{n}}{\xi-}+\frac{a}{n \omega_{Q}^{\prime}(\xi)}\right|^{n \beta_{n}} \\
& \quad=\exp \left(-n \beta_{n} \log \left|1+\frac{a}{n \omega_{Q}^{\prime}(\xi)\left(\xi-b_{n}\right)}\right|\right) \\
& \quad=\exp \left(n \beta_{n} O\left(\frac{R}{n \omega_{Q}^{\prime}(\xi)\left(\xi-b_{n}\right)}\right)\right)=\exp (o(1)),
\end{aligned}
$$

uniformly for $|a| \leq R$ and $\xi \in \mathcal{S}_{Q} \backslash \mathcal{E}_{n, 2}$, where again $\mathcal{E}_{n, 2}$ is a set of small measure, chosen so that $\omega_{Q}^{\prime}(\xi)$ is bounded above and below outside $\mathcal{E}_{n, 2}$, whereas a small interval around $b_{n}$ is excluded. Then (5.23) follows.

Finally, recall that $Q^{\prime}$ is assumed to be continuous except at finitely many points in $\Sigma$, whereas $\omega_{Q}^{\prime}$ is positive and continuous except at finitely many points. Taking $\mathcal{E}_{n, 3}$ consisting of small intervals centered on these points, we obtain a small set $\mathcal{E}_{n, 3}$ such that

$$
\begin{aligned}
& \sup _{\xi \in \mathcal{S}_{Q} \backslash \mathcal{E}_{n, 3},|a| \leq R} 2 n\left|Q(\xi)-Q\left(\xi+\frac{a}{n \omega_{Q}^{\prime}(\xi)}\right)\right| \\
& \leq 2 n \sup _{t \in \mathcal{S}_{Q} \backslash \mathcal{E}_{n, 3}}\left|Q^{\prime}(t)\right| \frac{R}{n \omega_{Q}^{\prime}(\xi)} \leq C<\infty .
\end{aligned}
$$

So we have (5.24).

Proof of Theorem 1.2. We apply Theorem 1.1 with

$$
\mu_{n}^{\prime}(x)=h(x) e^{-2 n Q(x)}
$$


and

where

$$
v_{n}^{\prime}(x)=g_{n}(x) \mu_{n}^{\prime}(x)
$$

$$
g_{n}(x)=e^{\tau_{n} h_{n}(x)}\left(\prod_{j=1}^{N}\left|x-\alpha_{n j}\right|^{\beta_{n j}}\right)^{n} .
$$

By a result of Totik [26, Theorem 1.2, p. 326], uniformly for $x$ in compact subsets of $\mathcal{S}_{Q}$ omitting discontinuities of $\omega_{Q}^{\prime}$, we have

$$
\lim _{n \rightarrow \infty} \frac{1}{n} K_{n}\left(\mu_{n}, x, x\right) \mu_{n}^{\prime}(x)=\omega_{Q}^{\prime}(x),
$$

so (1.9) is certainly true, and we can apply Lemma 2.2. Note also that by the aforementioned Theorem A of the authors [15, Theorem 1.1, p. 747],

$$
\lim _{n \rightarrow \infty} \frac{\tilde{K}_{n}\left(\mu_{n}, \xi+\frac{a}{\tilde{K}_{n}\left(\mu_{n}, \xi, \xi\right)}, \xi+\frac{b}{\tilde{K}_{n}\left(\mu_{n}, \xi, \xi\right)}\right)}{\tilde{K}_{n}\left(\mu_{n}, \xi, \xi\right)}=\mathbb{S}(a-b),
$$

uniformly for $\xi$ in compact subsets of $\mathcal{S}_{Q}$ omitting zeros of $Q^{\prime}$ and zeros or discontinuities of $\omega_{Q}^{\prime}$. We can replace $\tilde{K}_{n}\left(v_{n}, \xi, \xi\right)$ in this limit by $n \omega_{Q}^{\prime}(\xi)$ in view of Totik's asymptotics for Christoffel functions and the uniform convergence in $a, b$, giving, for the same range of $\xi, a, b$,

$$
\lim _{n \rightarrow \infty} \frac{\tilde{K}_{n}\left(\mu_{n}, \xi+\frac{a}{n \omega_{Q}^{\prime}(\xi)}, \xi+\frac{b}{n \omega_{Q}^{\prime}(\xi)}\right)}{n \omega_{Q}^{\prime}(\xi)}=\mathbb{S}(a-b) .
$$

Now let us turn to the construction of the polynomials $\left\{S_{n}\right\}$ in Theorem 1.1. We apply Lemmas 5.1 and 5.2. In applying Lemma 5.1, we choose $r$ sufficiently large and form a product of $N$ terms of the form $P_{n}\left(x-b_{n, j}\right)$, each with appropriately chosen $\delta_{j}$. We multiply the polynomials from Lemmas 5.1 and 5.2 to obtain $\left\{S_{n}\right\}$ satisfying (1.11)-(1.13). In fact, Lemma 5.1 and 5.2 give much more than (1.11)(1.13). Indeed, let $0<\eta<\frac{1}{2 N}$ and

$$
\mathcal{U}_{n}(\eta)=\bigcup_{j=1}^{N}\left[b_{n, j}-\eta, b_{n, j}+\eta\right],
$$

a set of measure $\leq 2 N \eta$. Lemmas 5.1 and 5.2 give polynomials $S_{n}$ of degree $o(n)$ such that, for each small enough $\eta$,

$$
\sup _{\xi \in \Sigma \backslash \mathcal{U}_{n}(\eta)}\left|\left(g_{n} S_{n}\right)(\xi)-1\right| \rightarrow 0, \quad n \rightarrow \infty .
$$

Note that also, given $R>0$ and $\eta>0$,

$$
\sup _{\xi \in \Sigma \backslash \mathcal{U}_{n}(\eta),|a| \leq R}\left|\left(g_{n} S_{n}\right)\left(\xi+\frac{a}{n}\right)-1\right| \rightarrow 0, \quad n \rightarrow \infty .
$$

Next, let us set

$$
\xi_{n, a}=\xi+\frac{a}{n \omega_{Q}^{\prime}(\xi)}
$$


with a similar notation for $\xi_{n, b}$. Also, let us replace $a, b$ in (4.7) by $a / \omega_{Q}^{\prime}(\xi)$ and $b / \omega_{Q}^{\prime}(\xi)$. We add to the set $\mathcal{E}_{n}$ in Theorem 4.3 small intervals centered on the zeros and discontinuities of $\omega_{Q}^{\prime}$. We obtain for $\xi \in \mathcal{S}_{Q} \backslash \mathcal{E}_{n}$ and all complex $a, b$,

$$
\begin{aligned}
& \frac{1}{n \omega_{Q}^{\prime}(\xi)}\left|\tilde{K}_{n}\left(v_{n}, \xi_{n, a}, \xi_{n, b}\right)-\left[\left(g_{n} S_{n}^{2}\right)\left(\xi_{n, a}\right)\left(g_{n} S_{n}^{2}\right)\left(\xi_{n, b}\right)\right]^{1 / 2} \tilde{K}_{n}\left(\mu_{n}, \xi_{n, a}, \xi_{n, b}\right)\right| \\
& \quad \times \frac{v_{n}^{\prime}(\xi)}{\left[v_{n}^{\prime}\left(\xi_{n, a}\right) v_{n}^{\prime}\left(\xi_{n, b}\right)\right]^{1 / 2}} \\
& \quad \leq \frac{8}{\sqrt{\varepsilon} \omega_{Q}^{\prime}(\xi)} \Gamma_{n}^{1 / 4} e^{C_{0}(|a|+|b|) / \varepsilon}
\end{aligned}
$$

Applying (5.26) and (5.27) and Lemma 5.3, we obtain for some suitable set $\mathcal{H}_{n}$ of small measure and for each $R>0$,

$$
\begin{aligned}
& \sup _{\xi \in \mathcal{S}_{Q} \backslash \mathcal{H}_{n},|a|,|b| \leq R}\left|\frac{1}{n \omega_{Q}^{\prime}(\xi)} \tilde{K}_{n}\left(v_{n}, \xi_{n, a}, \xi_{n, b}\right)-\mathbb{S}(a-b)\right| \\
& \leq \frac{C}{\sqrt{\varepsilon}} \sup _{\xi \in \Sigma \backslash \mathcal{H}_{n},|a|,|b| \leq R} \Gamma_{n}^{1 / 4} \frac{e^{C_{0} R / \varepsilon}}{\omega_{Q}^{\prime}(\xi)} \rightarrow 0, \quad n \rightarrow \infty .
\end{aligned}
$$

\section{References}

[1] G. Akemann, J. Baik, and P. Di Francesco, eds., The Oxford handbook of random matrix theory, Oxford University Press, Oxford, 2011.

[2] J. Baik, T. Kriecherbauer, K. T.-R. McLaughlin, and P. D. Miller, Uniform asymptotics for polynomials orthogonal with respect to a general class of discrete weights and universality results for associated ensembles, Princeton Annals of Mathematics Studies, 2006.

[3] C. Bennett and R. Sharpley, Interpolation of functions, Academic Press, Orlando, 1988.

[4] P. Bleher and A. Its, Random matrix models and their applications, Cambridge University Press, Cambridge, 2001.

[5] P. Deift, Orthogonal polynomials and random matrices: a Riemann-Hilbert approach, Courant Institute Lecture Notes, 3, New York University Pres, New York, 1999.

[6] P. Deift and D. Gioev, Random matrix theory: invariant ensembles and universality, Courant Institute Lecture Notes, 18, New York University Pres, New York, 2009.

[7] R. DeVore and G. G. Lorentz, Constructive approximation, Springer, Berlin, 1993.

[8] P. J. Forrester, Log-gases and random matrices, Princeton University Press, Princeton, 2010.

[9] A. Foulquie Moreno, A. Martinez-Finkelshtein, and V. Sousa, Asymptotics of orthogonal polynomials for a weight with a jump on $[-1,1]$, Constr. Approx. 33 (2011), 219-263.

[10] G. Freud, Orthogonal polynomials, Pergamon Press/Akademiai Kiado, Budapest, 1971.

[11] J. B. Garnett, Bounded analytic functions, Academic Press, Orlando, 1981.

[12] I. S. Gradshteyn and I. M. Ryzhik, Tables of integrals, series and products, Academic Press, San Diego, 1979. 
[13] A. B. Kuijlaars and M. Vanlessen, Universality for eigenvalue correlations from the modified Jacobi unitary ensemble, Int. Math. Res. Not. 30 (2002), 1575-1600.

[14] E. Levin and D. S. Lubinsky, Orthogonal polynomials for exponential weights, Springer, New York, 2001.

[15] _ Universality limits in the bulk for varying measures, Adv. Math. 219 (2008), 743-779.

[16] _ Universality limits for exponential weights, Constr. Approx. 29 (2009), 247275.

[17] D. S. Lubinsky, A new approach to universality limits involving orthogonal polynomials, Ann. Math. 170 (2009), 915-939.

[18] _ Bulk universality holds in measure for compactly supported measures, J. Anal. Math. 116 (2012), 219-253.

[19] L. Pastur and M. Shcherbina, Universality of the local eigenvalue statistics for a class of unitary invariant random matrix ensembles, J. Stat. Phys. 86 (1997), 109-147.

[20] T. Ransford, Potential theory in the complex plane, Cambridge University Press, Cambridge, 1995.

[21] W. Rudin, Real and complex analysis, Wiley, McGraw Hill, Singapore, 1987.

[22] E. B. Saff and V. Totik, Logarithmic potentials with external fields, Springer, New York, 1997.

[23] B. Simon, Orthogonal polynomials on the unit circle, parts 1 and 2, American Mathematical Society, Providence, 2005.

[24] , Two extensions of Lubinsky's universality theorem, J. Anal. Math. 105 (2008), 345-362.

[25] V. Totik, Weighted approximation with varying weight, Lecture Notes in Math., 1569, Springer, Berlin, 1994.

[26] - Asymptotics for Christoffel functions with varying weights, Adv. in Appl. Math. 25 (2000), 322-351.

[27] _ Universality and fine zero spacing on general sets, Ark. Mat. 47 (2009), 361-391.

[28] _ Universality under Szegố's condition, Canad. Math. Bull. 59 (2016), 211224.

E. Levin

Mathematics Department

The Open University of Israel

P.O. Box 808

Raanana 43107

Israel

elile@openu.ac.il
D. S. Lubinsky

School of Mathematics

Georgia Institute of Technology

Atlanta, GA 30332-0160

USA 